\title{
Manejo de pacientes con enfermedad inflamatoria intestinal durante la pandemia de COVID-19
}

\author{
Management of patients with inflammatory bowel disease during the \\ COVID-19 pandemic
}

Fabián Juliao-Baños. ${ }^{*}$

1 Unidad de Gastroenterología, Coordinador de la Clínica de Enfermedad Inflamatoria Intestinal,

Hospital Pablo Tobón Uribe. Medellín, Colombia.

*Correspondencia: Fabían Juliao-Baños

fabianjuliao@hotmail.com

\begin{abstract}
Resumen
La pandemia causada por el virus SARS-CoV-2, originada en Wuhan, capital de la provincia de Hubei (China), se ha convertido en un desafio para la humanidad. Es un virus altamente contagioso; hasta el momento, la enfermedad por coronavirus (COVID-19) presenta una mortalidad global alrededor del $6 \%$, que es mayor en pacientes con comorbilidades. La enfermedad inflamatoria intestinal (EII) es una patología discapacitante, con daño tisular y alteración en la respuesta inmunológica, lo cual, asociado a los medicamentos inmunosupresores que se utilizan frecuentemente para su tratamiento, pone al paciente en riesgo de desarrollar infecciones y complicaciones. En esta revisión consideramos la interacción del virus SARS-CoV-2 con el tracto gastrointestinal y los potenciales mecanismos por los cuales un paciente con Ell podría tener un riesgo incrementado de la infección por COVID-19. Adicionalmente, y a pesar de que no hay estudios clínicos publicados en pacientes con Ell y COVID-19, damos recomendaciones basadas en opinión de expertos sobre el cuidado de pacientes con Ell, con énfasis en su tratamiento y la realización segura de procedimientos endoscópicos, tanto para el paciente como para el personal de salud.
\end{abstract}

\section{Palabras clave}

COVID-19, SARS-CoV-2, enfermedad inflamatoria intestinal.

\begin{abstract}
The pandemic caused by the SARS-Cov-2 virus originating in Wuhan, capital of the province of Hubei (China), has become a challenge for humanity. It is a highly contagious virus and up to now the COVID-19 disease has an overall mortality of around $6 \%$, which is higher in patients with comorbidities. Inflammatory bowel disease (IBD) is a disabling pathology, with tissue damage and impaired immune response, which, associated with immunosuppressive drugs that are frequently used for their treatment, put the patient at risk of developing infections and complications. In this review we consider the interaction of the SARS-CoV-2 virus with the gastrointestinal tract and the potential mechanisms whereby a patient with IBD could have an increased risk of COVID-19 infection. Additionally, and despite the fact that there are no published clinical studies in patients with IBD and COVID-19, we make recommendations based on the opinion of experts on the care of patients with IBD, with an emphasis on its treatment and the safe performance of endoscopic procedures, both for the patient and the health personnel.
\end{abstract}

\section{Keywords}

COVID-19, SARS-CoV-2, inflammatory bowel disease. 


\section{INTRODUCCIÓN}

COVID-19 es una enfermedad producida por el coronavirus SARS-CoV-2, un virus ARN cuyo nombre deriva de la morfología de su envoltura en forma de "corona"; este se originó en Wuhan, capital de la provincia de Hubei (China), desde diciembre de 2019 (1). El 7 de enero de 2020 fue aislado en muestras del tracto respiratorio de 4 pacientes, en las que se encontró que es un poco diferente y más contagioso que las otras dos cepas de coronavirus causantes de epidemias previas, el SARS-CoV (severe acute respiratory syndrome) y el MERS-CoV (Middle East respiratory syndrome) (2).

El SARS-CoV-2 se ha diseminado por todo el mundo, por lo cual el 11 de marzo de 2020 la Organización Mundial de la Salud (OMS) declaró la pandemia (3). Para el 15 de abril de 2020, tenemos 185 países afectados, con 2049888 personas infectadas en el mundo y 133572 muertos, lo que da una mortalidad global de 6,5\% (4).

La ruta principal de contaminación es por microgotas de saliva y partículas aerosolizadas $(<5 \mu \mathrm{m})$ cargadas de virus y que se desplazan por el aire; sin embargo, tocar superficies contaminadas con dichas partículas y tener contacto persona a persona también pueden transmitir el virus. Se ha demostrado que el virus SARS-CoV-2 puede permanecer viable en superficies como plásticos y acero inoxidable hasta por 72 horas (5). El promedio de incubación es de 5,1 días (intervalo de confianza [IC] 95\%, 4,5-5,8 días), y el 97,5\% de los pacientes desarrollan síntomas en promedio dentro de 11,5 días (IC $95 \%$, 8,2-15,6 días) posterior al contagio (6). En un estudio en China, se documentó la presencia del SARSCoV-2 en la materia fecal en el $53 \%$ de 73 pacientes hospitalizados con COVID-19, lo que abre la posibilidad de una transmisión oro-fecal, aunque esto aún no ha sido demostrado (7). El primer caso en Estados Unidos se detectó en el estado de Washington el 20 de enero de 2020; el paciente presentó síntomas gastrointestinales como náuseas, vómito, diarrea y molestia abdominal, y se le realizó una prueba para SARS-Cov-2 en materia fecal al séptimo día del comienzo de la infección, la cual fue positiva (8).

Las manifestaciones clínicas de COVID-19 son variables. Un reciente metaanálisis de 60 estudios con 59254 pacientes de 11 países encontró que los síntomas más frecuentes son: fiebre $(82 \%)$, tos seca $(61 \%)$, fatiga y mialgias $(36 \%)$, disnea $(26 \%)$, cefalea $(12 \%)$, dolor de garganta $(10 \%)$ y síntomas gastrointestinales ( $9 \%)$ (9). En cuanto al espectro clínico de COVID-19 en 44672 casos confirmados en China, la mayoría (75\%) proveniente de la provincia de Hubei, fue el siguiente: $81 \%$ fueron leves (sin neumonía o neumonía leve), $14 \%$ con neumonía grave (disnea, frecuencia respiratoria $\geq 30$ respiraciones/min, saturación de oxígeno en sangre $\leq 93 \%$, presión parcial de oxígeno arterial a fracción de oxígeno inspirado $<300$, y/o infiltrados pulmonares $>50 \%$ en $24-48$ horas), y $5 \%$ críticos (falla respiratoria, choque séptico, y/o disfunción multiorgánica) (10).

En otro metaanálisis de 60 estudios con 4243 pacientes se encontraron síntomas gastrointestinales en $17,6 \%$ de individuos (IC $95 \%, 12,3 \%-24,5 \%$ ); en aquellos con COVID19 no graves, el 11,8 \% (IC $95 \%, 4,1 \%-29,1 \%$ ) presentaban síntomas gastrointestinales, mientras que $17,1 \%$ (IC $95 \%, 6,9 \%-36,7 \%$ ) de individuos con COVID-19 grave tenían síntomas gastrointestinales. La prevalencia del SARS-CoV-2 en muestras de materia fecal fue de $48,1 \%$ (IC $95 \%$, 38,3 \%-57,9 \%). De estas muestras, el 70,3\% fueron recolectadas después de tener resultados negativos en las pruebas de tracto respiratorio (11).

\section{¿EXISTE UN MAYOR RIESGO DE INFECCIÓN POR SARS-COV-2 EN PACIENTES CON ENFERMEDAD INFLAMATORIA INTESTINAL?}

La enfermedad inflamatoria intestinal (EII) comprende dos entidades: la colitis ulcerativa (CU) y la enfermedad de Crohn (EC), las cuales son patologías inflamatorias crónicas del tracto gastrointestinal, poco comunes, que afectan primordialmente el colon y el intestino delgado, y cuya etiología es multifactorial $(12,13)$. Su curso clínico se caracteriza por presentar múltiples recaídas; durante los últimos años, se ha detectado un aumento en la frecuencia de aparición tanto a nivel mundial como en Latinoamérica $(14,15)$. La EII es una enfermedad discapacitante, con daño tisular y alteración en la respuesta inmunológica, lo cual, asociado a los medicamentos que se utilizan para su tratamiento, ponen al paciente en riesgo de desarrollar infecciones y complicaciones (16-19).

Los pacientes con EII pueden, hipotéticamente, tener un riesgo incrementado de COVID-19. El receptor del SARS$\mathrm{CoV}-2$ en la célula blanco es el receptor tipo 2 de la enzima convertidora de angiotensina (ECA), y necesita también a la proteasa TMPRSS2 para poder entrar a la célula. El receptor de la ECA tipo 2, además de expresarse en el tracto respiratorio superior, los pulmones (neumocitos tipo II), el corazón, los vasos sanguíneos y el riñón, se encuentra en el tracto digestivo, con una mayor concentración en el esófago, el íleon terminal y en el colon $(7,20)$. Lo anterior podría explicar el alto porcentaje del SARS-CoV-2 positivo en heces y los síntomas gastrointestinales asociados a esta infección $(7,11,21)$. Adicionalmente, se ha documentado un incremento en la expresión del receptor de ECA tipo 2 en el intestino inflamado de pacientes con EII (22). Todas estas observaciones sugieren que el intestino inflamado de los pacientes con EII puede facilitar la entrada del SARSCoV-2 en el organismo; sumado a esto, los pacientes con EII reciben medicamentos que se asocian con un incre- 
mento en el riesgo de infecciones (16-19). A pesar de lo anterior, hasta el momento no hay estudios publicados en pacientes con EII y COVID-19; por lo tanto, no se pueden hacer recomendaciones específicas basadas en evidencia clínica directa (23).

Recientemente fue creado un registro internacional de pacientes con EII denominado SECURE-IBD (Surveillance Epidemiology of Coronavirus Under Research Exclusion Inflammatory bowel disease), que incluye tanto adultos como niños y sirve para monitorizar y mostrar resultados de COVID-19 en individuos con EII. Para abril 15 de 2020 se han registrado 525 casos en 35 países del mundo, 33,5\% provienen de Estados Unidos y 16,7 \% de España, con una mortalidad de $2 \%$ en EC y $5 \%$ en CU, la cual es mayor en hombres ( $4 \%$ frente a $2 \%$ ), en fumadores ( $4 \%$ frente a $3 \%$ ) y en pacientes $>60$ años o con comorbilidades. La mortalidad observada en individuos tratados con ácido 5-aminosalicílico (5-ASA) es de $8 \%$, budesonida $6 \%$, esteroides $11 \%$, azatioprina $2 \%$, metotrexato $0 \%$, monoterapia con un antagonista del factor de necrosis tumoral alfa (antiFNT $\alpha$ ) $1 \%$, terapia combinada con inmunosupresores $4 \%$, anti-integrinas $0 \%$, y anti-interleucina (IL)-12/23 $0 \%$ (24). A pesar de las tendencias presentadas, se debe esperar tener resultados con un mayor número de pacientes para sacar conclusiones definitivas de este interesante registro.

\section{RECOMENDACIONES PARA PACIENTES CON ENFERMEDAD INFLAMATORIA INTESTINAL DURANTE LA PANDEMIA POR COVID-19}

La pandemia por COVID-19 ha tenido una diseminación muy rápida, y aún se encuentra en evolución; por lo tanto, aún no están disponibles datos amplios de incidencia o factores de riesgo en pacientes con EII. El centro de EII de la Universidad Humanitas de Milán (Italia) (25) y, más recientemente, la Organización Internacional para el estudio de EII (IOIBD) (26), teniendo en cuenta que COVID-19 no es una infección oportunista y que es extremadamente contagiosa, sugieren seguir las mismas instrucciones de medidas protectoras básicas recomendadas por la OMS para la población general en los pacientes con EII (27):

- Limpie sus manos regularmente y a fondo con un desinfectante para manos a base de alcohol, o lávelas con agua y jabón.

- Limpie las superficies con un desinfectante a base de alcohol dónde crea que pueda haber gotas de saliva.

- Mantenga al menos 1 metro de distancia de cualquier persona que esté tosiendo o estornudando.

- Evite tocarse los ojos, la nariz y la boca (un tapabocas puede ayudar en esto).

- Asegúrese de que usted y las personas que lo rodean sigan una buena higiene respiratoria. Esto significa cubrirse la boca y la nariz con el codo o un pañuelo doblado cuando tosa o estornude.

- Quédese en casa si no se siente bien. Si tiene fiebre, tos y dificultad para respirar, busque atención médica.

- Use un tapabocas para evitar infectar a otras personas, incluso en caso de que presente síntomas leves.

- Use guantes cuando vaya de compras, cuando use la bomba de gasolina, y en todas las demás actividades externas que impliquen riesgo de contaminación de manos.

- Evite usar baños públicos tanto como sea posible, ya que el inodoro, el lavamanos y la manija de la puerta pueden estar contaminados.

- Manténgase actualizado sobre los últimos sitios críticos de COVID-19. Si es posible, evite viajes no urgentes, especialmente si es una persona mayor o tiene diabetes, enfermedades cardíacas o pulmonares.

Adicionalmente, en el centro de EII Humanitas en Italia considera que mantener la comunicación con los pacientes es fundamental, ya sea por correo electrónico o por vía telefónica, y que el papel de la enfermera es clave. Se les insiste a los pacientes continuar con su mismo tratamiento, sobre todo si están en remisión, y seguir las recomendaciones de la asociación nacional de pacientes de Italia. Además, durante esta pandemia, la farmacia se encarga de suministrar medicamentos a domicilio por un período de hasta 4 meses, incluyendo aquellos de aplicación subcutánea para administrar en casa (28).

\section{RECOMENDACIONES PARA LOS CENTROS DE ENFERMEDAD INFLAMATORIA INTESTINAL DURANTE LA PANDEMIA POR COVID-19}

El centro de EII de la Universidad Humanitas de Milán (Italia) atiende más de 5000 pacientes de todo el país y, por la pandemia COVID-19, se ha convertido en una clínica de atención virtual; las cirugías electivas fueron aplazadas, y solamente se manejan en forma presencial los casos clínicos y quirúrgicos urgentes, $\mathrm{u}$ oncológicos, lo cual tiene como desventaja una posible progresión de la enfermedad, y resultados quirúrgicos quizá no tan favorables por aplazar dichos procedimientos. Toda esta situación genera ansiedad en los pacientes, pero hay que insistirles en que esta pandemia es temporal, y que es necesario que se mantengan en contacto con el centro para suministrarles todo el soporte necesario (28).

Todos los trabajadores de la salud y los pacientes son interrogados a la entrada del hospital; se les pregunta por síntomas como tos y fiebre en las últimas dos semanas, o si han tenido contacto cercano con familiares con estos síntomas, o conocidos infectados por SARS-CoV-2. Los pacien- 
tes que ingresan para procedimientos de estudios clínicos o para infusión de medicamentos son atendidos en forma presencial, pero sin acompañantes, y las sillas para infusión de medicamentos están separadas a una distancia segura (1-2 metros) (28). A los pacientes se les coloca tapabocas quirúrgico, y todo el personal de salud cuenta con equipo de protección personal (EPP) y siguen las recomendaciones de la OMS anteriormente descritas (27). El uso de esteroides en la infección por COVID-19 es controversial (29), razón por la cual intentan usar dosis bajas ( $<20 \mathrm{mg} /$ día $)$ y por corto tiempo solo en caso de recaídas. Adicionalmente, sugieren evitar el inicio de terapia biológica durante esta pandemia en pacientes asintomáticos, por ejemplo, en la prevención de recurrencia posquirúrgica en EC de riesgo bajo a moderado, y tampoco recomiendan cambiar de terapia biológica de presentación intravenosa a una subcutánea en pacientes en remisión (25).

Hasta el momento, no hay recomendaciones basadas en evidencia clínica durante esta pandemia por COVID-19 para pacientes en inmunosupresión, como es el caso de la EII. La IOIBD está conformada por 89 miembros expertos en EII de 26 diferentes países del mundo, y ha publicado una serie de recomendaciones basadas en la opinión de expertos por medio de votación virtual el día 27 de marzo de 2020, relacionadas con el riesgo de infección por el SARS-CoV-2, el desarrollo de COVID-19, y el manejo de la EII en diferentes escenarios clínicos. A cada declaración se le daba un puntaje de 1 a 9: un puntaje promedio de 1 a 3 se consideró no apropiada, de 4 a 6 incierta y de 7 a 9 apropiada. En total se votaron 76 declaraciones, de las cuales 26 fueron apropiadas, 19 inciertas y 31 no apropiadas (26). Se consideró apropiada la declaración de que no hay riesgo adicional de contraer la infección por el virus SARS-CoV-2 en pacientes con EII en comparación con la población general, y que no existen diferencias en cuanto al riesgo de pacientes con EC y $\mathrm{CU}$; sin embargo, consideran incierta la posibilidad de que los pacientes con EII activa con inflamación puedan tener mayor riesgo de infección por el SARS-CoV-2.

Adicionalmente, el panel consideró apropiado aplazar procedimientos endoscópicos y quirúrgicos no urgentes, suspender viajes no esenciales, continuar con el tratamiento inmunosupresor en pacientes con EII si se encuentran en remisión; si están en terapia combinada (anti-FNT más inmunosupresor) y contraen la infección por el SARSCoV-2, pero no desarrollan COVID-19, se debe suspender el inmunosupresor. A pesar de que las tiopurinas se han asociado a infecciones virales (17-19), el panel considera continuarlas, ya que suspenderlas no ayudaría a corto plazo debido a que tienen una vida media larga de eliminación. En caso de suspender el medicamento por contraer COVID-19, se debe reiniciar después de 14 días cuando el paciente se encuentre asintomático, y después de dos muestras negativas de nasofaringe para el SARS-CoV-2. A los pacientes con EII asintomáticos que se infecten por el virus, pero no desarrollen COVID-19, se les debe reiniciar el tratamiento a los 14 días. Un resumen de las declaraciones y votaciones del panel sobre las opciones de tratamiento de la EII y diferentes escenarios clínicos de COVID-19 se presenta en la Tabla 1.

\section{RECOMENDACIONES PARA LOS PROCEDIMIENTOS ENDOSCÓPICOS EN PACIENTES CON ENFERMEDAD INFLAMATORIA INTESTINAL DURANTE LA PANDEMIA POR COVID-19}

La transmisión oro-fecal puede ser parte del espectro clínico de la infección por SARS-Cov-2 ante la evidencia disponible $(7,30,31)$. El ARN de este virus se elimina en las heces y puede persistir hasta incluso después de que desaparezcan los síntomas respiratorios (11). No obstante, una publicación reciente muestra que, aunque se pudieron detectar grandes cantidades de ARN viral de SARS-CoV-2 en las heces, no se pudo replicar el virus en dichas muestras de materia fecal (32).

La principal ruta de transmisión de COVID-19 de humano a humano es la vía aérea, y aunque existe la posibilidad de transmisión fecal, esto no se ha demostrado; por lo tanto, ante la corta distancia física que existe entre los pacientes y el personal durante los procedimientos endoscópicos, se considera que tanto las endoscopias superiores como las inferiores pueden conllevar un riesgo de infección por el SARS-CoV-2. Diversas sociedades científicas han publicado recomendaciones sobre procedimientos endoscópicos durante la pandemia por COVID-19, las cuales se pueden aplicar a los pacientes con EII (33-37). La IOIBD considera apropiado aplazar todo procedimiento endoscópico y quirúrgico no urgente en los pacientes con EII (26). Siguiendo las anteriores recomendaciones, en nuestro hospital, el Pablo Tobón Uribe en Medellín (Colombia), limitamos la realización de estudios endoscópicos a pacientes con EII que requieran procedimientos urgentes (hemorragia digestiva con inestabilidad hemodinámica, colangitis aguda, extracción de cuerpo extraño, obstrucción del tubo digestivo y a pacientes con cáncer). Decidimos entonces estratificar el riesgo del procedimiento endoscópico a realizar (Tabla 2), y definimos el riesgo de cada paciente según los síntomas que presente y los antecedentes que tenga (Tabla 3); basados en lo anterior, determinamos el nivel de protección del personal de salud para cada procedimiento endoscópico (Tabla 4). 
Tabla 1. Posición de la IOIBD sobre el uso de medicamentos en EII y COVID-19 (26).

\begin{tabular}{|c|c|c|c|c|c|c|c|c|c|}
\hline Declaración & 5-ASA & BUD & $\begin{array}{l}\text { Prednisolona } \\
\text { (>20 mg/día) }\end{array}$ & AZA/6MP & MTX & Anti-FNT & VEDO & UST & TOFA \\
\hline $\begin{array}{l}\text { Esta terapia incrementa el riesgo de infección por } \\
\text { SARS-CoV-2 }\end{array}$ & $\mathrm{N}$ & $\mathrm{N}$ & $A$ & I & I & I & $\mathrm{N}$ & $\mathrm{N}$ & I \\
\hline Esta terapia incrementa el riesgo de COVID-19 & $\mathrm{N}$ & $\mathrm{N}$ & A & I & I & I & $\mathrm{N}$ & $\mathrm{N}$ & I \\
\hline $\begin{array}{l}\text { Pacientes con esta terapia deben reducir la dosis } \\
\text { para prevenir infección por SARS-CoV-2 }\end{array}$ & $\mathrm{N}$ & $\mathrm{N}$ & A & $\mathrm{N}$ & $\mathrm{N}$ & $\mathrm{N}$ & $\mathrm{N}$ & $\mathrm{N}$ & $\mathrm{N}$ \\
\hline $\begin{array}{l}\text { Pacientes con esta terapia deben suspenderla para } \\
\text { prevenir infección por SARS-CoV-2 }\end{array}$ & $\mathrm{N}$ & $\mathrm{N}$ & A & $\mathrm{N}$ & $\mathrm{N}$ & $\mathrm{N}$ & $\mathrm{N}$ & $\mathrm{N}$ & $\mathrm{N}$ \\
\hline $\begin{array}{l}\text { Pacientes con esta terapia deben suspenderla si son } \\
\text { positivos para SARS-CoV-2 pero no tienen COVID-19 }\end{array}$ & $\mathrm{N}$ & I & $A$ & $A$ & $A$ & 1 & I & I & $A$ \\
\hline $\begin{array}{l}\text { Pacientes con esta terapia deben suspenderla si } \\
\text { desarrollan COVID-19 }\end{array}$ & $\mathrm{N}$ & I & $A$ & $A$ & $A$ & $A$ & 1 & $A$ & $A$ \\
\hline
\end{tabular}

A: apropiado; N: no apropiado; I: incierto; Anti-FNT: antifactor de necrosis tumoral; AZA: Azatioprina; BUD: budesónida; MTX: metotrexato; TOFA: tofacitinib; UST: ustekinumab; VEDO: vedolizumab; 6MP: 6-mercaptopurina. Adaptado de referencia 26.

Tabla 2. Nivel de riesgo según el paciente

\begin{tabular}{lll}
\hline \multicolumn{1}{c}{ Riesgo bajo } & \multicolumn{1}{c}{ Riesgo intermedio } & \multicolumn{1}{c}{ Riesgo alto } \\
\hline Sin síntomas & Presencia de síntomas con: & Mínimo 1 síntoma + 1 de los siguientes: \\
& - Sin contacto con alguien positivo para SARS-CoV-2 & - Contacto con alguien positivo para SARS-CoV-2 \\
& - No procede de áreas de alto riesgo en los 14 días previos & - Procede de zona de alto riesgo en los 14 días previos \\
Sin contacto con alguien & Sin síntomas, pero: & \\
positivo para SARS-CoV-2 & - Contacto con alguien positivo para SARS-CoV-2 & \\
& - Procede de áreas de alto riesgo en los 14 días previos & \\
$\begin{array}{l}\text { No procede de áreas de } \\
\text { alto riesgo en los 14 días } \\
\text { previos }\end{array}$ & \\
\hline
\end{tabular}

Tabla 3. Nivel de riesgo según el tipo de endoscopia

Endoscopias de riesgo intermedio

Endoscopia baja (incluye colonoscopia, ecoendoscopia baja)

\section{Endoscopias de alto riesgo}

Endoscopia alta y broncoscopia (incluye gastroscopias, colangiopancreatografía retrógrada endoscópica [CPRE], ecoendoscopias altas)

\section{CONCLUSIONES}

La pandemia por COVID-19 se ha convertido en un gran problema para la humanidad; hasta el momento, no hay recomendaciones basadas en evidencia clínica para pacientes con EII. Existe una interacción de este virus con el tracto gastrointestinal a través de la interacción con el receptor de la ECA tipo 2, y se ha documentado su eliminación fecal, aunque la transmisión por esta vía no se ha demostrado. Existen recomendaciones basadas en la opinión de expertos sobre el cuidado de pacientes con EII durante la pandemia por COVID-19, teniendo en cuenta el riesgo de la infección y su tratamiento $(26,38)$. En la Figura 1 se propone un algoritmo de manejo de pacientes con EII y dife- 
Tabla 4. Nivel de protección según el riesgo del paciente y del procedimiento

\begin{tabular}{|c|c|}
\hline Paciente de riesgo intermedio & Paciente de riesgo alto \\
\hline $\begin{array}{l}\text { Endoscopia baja } \\
\text { - Careta de protección } \\
\text { - Guantes } \\
\text { - Gorro } \\
\text { - Bata impermeable o bata de un solo uso, más delantal encima } \\
\text { - Mascarillas quirúrgicas }\end{array}$ & $\begin{array}{l}\text { Cualquier endoscopia } \\
\text { - Careta de protección } \\
\text { - Guantes } \\
\text { - Gorro } \\
\text { - Bata impermeable o bata de un solo uso, más delantal encima } \\
\text { - Mascarilla N95 más careta }\end{array}$ \\
\hline $\begin{array}{l}\text { Endoscopia alta } \\
\text { - Careta de protección } \\
\text { - Guantes } \\
\text { - Gorro } \\
\text { - Bata impermeable o bata de un solo uso más delantal encima } \\
\text { - Mascarillas N95 más careta }\end{array}$ & \\
\hline
\end{tabular}

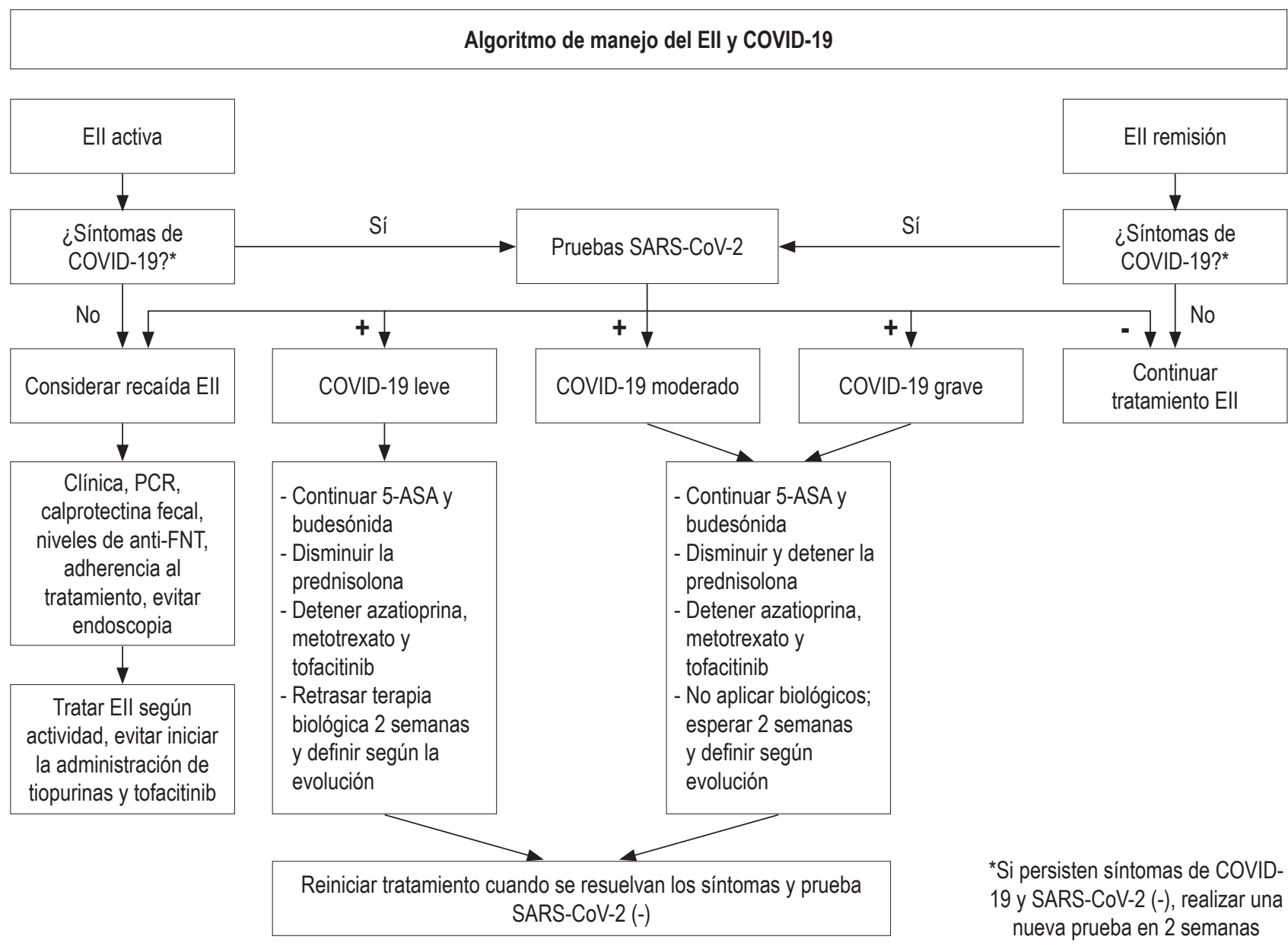

Figura 1. Algoritmo de manejo EII y COVID-19. Adaptado de la referencia 38. 
rentes escenarios clínicos de la infección por SARS-CoV-2 y el desarrollo de COVID-19.

Dado que la pandemia por COVID-19 es dinámica y el conocimiento está creciendo rápidamente, algunas de estas recomendaciones presentadas en esta revisión pueden actualizarse de acuerdo con la aparición de mejor evidencia clínica. Se esperan resultados definitivos del registro SECURE-IBD.

\section{Fuente de financiación}

Para la realización de este estudio, no se recibió financiación de ninguna entidad.

\section{REFERENCIAS}

1. Lu R, Zhao X, Li J, Niu P, Yang B, Wu H, et al. Genomic characterization and epidemiology of 2019 novel coronavirus: implication for virus origins and receptor binding. Lancet 2020;395:565-574. https://doi.org/10.1016/S01406736(20)30251-8

2. Zhu N, Zhang D, Wang W, Li X, Yang B, Song J, et al. A Novel Coronavirus from Patients with Pneumonia in China, 2019. N Engl J Med. 2020;382(8):727-733. https://doi. org/10.1056/NEJMoa2001017

3. World Health Organization [Internet]. Coronavirus. 2020 [acceso 15 de abril de 2020]. Disponible en: https://www. who.int/health-topics/coronavirus

4. Johns Hopkins University Coronavirus Resource Center [Internet]. Coronavirus COVID-19 Global Cases 2020 [acceso 15 de abril de 2020]. Disponible en: https://coronavirus.jhu.edu/map.html

5. van Doremalen N, Bushmaker T, Morris DH, Holbrook MG, Gamble A, Williamson BN, et al. Aerosol and Surface Stability of SARS-CoV-2 as Compared with SARS-CoV-1. N Engl J Med. 2020;382(16):1564-1567. https://doi. org/10.1056/NEJMc2004973

6. Lauer SA, Grantz KH, Bi Q Jones FK, Zheng Q, Meredith HR, et al. The Incubation Period of Coronavirus Disease 2019 (COVID-19) From Publicly Reported Confirmed Cases: Estimation and Application. Ann Intern Med. 2020; M200504. https://doi.org/10.1101/2020.02.02.20020016

7. Xiao F, Tang M, Zheng X, Liu Y, Li X, Shan H. Evidence for gastrointestinal infection of SARSCoV-2. Gastroenterology. 2020; S0016508520302821. https://doi.org/10.1053/j. gastro.2020.02.055

8. Holshue ML, DeBolt C, Lindquist S, Lofy KH, Wiesman J, Bruce H, et al. First Case of 2019 Novel Coronavirus in the United States. N Engl J Med. 2020;382(10):929-936. https://doi.org/10.1056/NEJMoa2001191

9. Borges do Nascimento IJ, Cacic N, Abdulazeem HM, Caspar von Groote T, Jayarajah U, Weerasekara I, et al. Novel Coronavirus Infection (COVID-19) in Humans: A Scoping Review and Meta-Analysis. J Clin Med. 2020;9(4):E941. https://doi.org/10.3390/jcm9040941
10. $\mathrm{Wu} \mathrm{Z}$, McGoogan JM. Characteristics of and Important Lessons from the Coronavirus Disease 2019 (COVID19) Outbreak in China: Summary of a Report of 72314 Cases from the Chinese Center for Disease Control and Prevention. JAMA. 2020. https://doi.org/10.1001/ jama.2020.2648

11. Cheung KS, Hung I, Chang P, Lung KC, Tso E, Liu R, et al. Gastrointestinal Manifestations of SARS-CoV-2 Infection and Virus Load in Fecal Samples from the Hong Kong Cohort and Systematic Review and Meta-analysis. 2020. https://doi.org/10.1053/j.gastro.2020.03.065

12. Ungaro R, Mehandru S, Allen PB, Peyrin-Biroulet L, Colombel J-F. Ulcerative Colitis. Lancet. 2017;389(10080):1756-1770. https://doi.org/10.1016/ S0140-6736(16)32126-2

13. Torres J, Mehandru S, Colombel JF, Peyrin-Biroulet L. Crohn's Disease. Lancet. 2017;389(10080):1741-1755. https://doi.org/10.1016/S0140-6736(16)31711-1

14. Ng SC, Shi HY, Hamidi N, Underwood FE, Whitney Tang W, Benchimol EL, et al. Worldwide incidence and prevalence of inflammatory bowel disease in the 21st century: a systematic review of population-based studies. Lancet. 2018;390(10114):2769-2778. https://doi.org/10.1016/ S0140-6736(17)32448-0

15. Kotze PG, Underwood F, Damiao AOMC, Ferraz JG, Saad-Hossne R, Toro M, et al. Progression of Inflammatory Bowel Diseases Throughout Latin America and the Caribbean: A Systematic Review. Clin Gastroenterol Hepatol. 2020;18(2):304-312. https://doi.org/10.1016/j. cgh.2019.06.030

16. Bonovas S, Fiorino G, Allocca M, Lytras T, Nikolopoulos GK, Peyrin-Biroulet L, et al. Biologic Therapies and Risk of Infection and Malignancy in Patients with Inflammatory Bowel Disease: A Systematic Review and Network Metaanalysis. Clin Gastroenterol Hepatol. 2016;14(10):13851397. https://doi.org/10.1016/j.cgh.2016.04.039

17. Govani SM, Higgins PD. Combination of thiopurines and allopurinol: adverse events and clinical benefit in IBD. J Crohns Colitis. 2010;4(4):444-9. https://doi. org/10.1016/j.crohns.2010.02.009

18. Wisniewski A, Kirchgesner J, Seksik Ph, Landman C, Bourrier A, Nion-Larmurier I, et al. Increased incidence of systemic serious viral infections in patients with inflammatory bowel disease associates with active disease and use of thiopurines. United Eur. Gastroenterol. J. 2020;8(3):303313. https://doi.org/10.1177/2050640619889763

19. Toruner M, Loftus EV, Harmsen WS, Zinsmeister AR, Orenstein R, Sandborn WJ, et al. Risk factors for opportunistic infections in patients with inflammatory bowel disease. Gastroenterology 2008;134(4):929-936. https:// doi.org/10.1053/j.gastro.2008.01.012

20. Hoffmann M, Kleine-Weber H, Schroeder S, Krüger N, Herrler T, Erichsen S, et al. SARS-CoV-2 Cell Entry Depends on ACE2 and TMPRSS2 and Is Blocked by a Clinically Proven Protease Inhibitor. Cell. 2020. https:// doi.org/10.1016/j.cell.2020.02.052 
21. Monteleone G, Ardizzone S. Are patients with inflammatory bowel disease at increased risk for Covid-19 infection? J Crohns Colitis. 2020. https://doi.org/10.1093/ecco-jcc/ jjaa061

22. Garg M, Royce SG, Tikellis C, Shallue C, Batu D, Velkoska $\mathrm{E}$, et al. Imbalance of the renin-angiotensin system may contribute to inflammation and fibrosis in IBD: a novel therapeutic target? Gut. 2020;69(5):841-851. http://dx.doi. org/10.1136/gutjnl-2019-318512

23. Mao R, Liang J, Shen J, Ghosh S, Zhu LR, Yang H, et al. Implications of COVID-19 for patients with pre-existing digestive diseases. Lancet Gastroenterol Hepatol. 2020;5(5):426428. https://doi.org/10.1016/S2468-1253(20)30076-5

24. Brenner EJ, Ungaro RC, Colombel JF, Kappelman MD. Surveillance Epidemiology of Coronavirus) Under Research Exclusion (SECURE-IBD) [acceso 15 de abril de 2020]. Database Public Data Update. Disponible en: https: / / www. covidibd.org

25. Fiorino G, Allocca M, Furfaro F, et al. Inflammatory bowel disease care in the COVID-19 pandemic era: the Humanitas, Milan experience. J Crohns Colitis. 2020. https://doi. org/10.1093/ecco-jcc/jjaa058

26. Rubin DT, Abreu MT, Rai V, Siegel CA; International Organization for the Study of Inflammatory Bowel Disease. Management of Patients with Crohn's Disease and Ulcerative Colitis During the COVID-19 Pandemic: Results of an International Meeting. Gastroenterology. 2020; S0016-5085(20)30465-0. https://doi.org/10.1053/j.gastro.2020.04.002

27. World Health Organization [Internet]. Q\&A on Coronaviruses [COVID-19]. [ acceso 15 de abril de 2020]. Disponible en: https://www.who.int/news-room/q-adetail/q-a-coronaviruses

28. Danese S, Cecconi M, Spinelli A. Management of IBD during the COVID-19 outbreak: resetting clinical priorities. Nat Rev Gastroenterol Hepatol. 2020. https://doi. org/10.1038/s41575-020-0294-8

29. Shang L, Zhao J, Hu Y, Du R, Cao B. On the use of corticosteroids for 2019-nCoV pneumonia. Lancet. 2020; 395:6834. https://doi,org/10.1016/S0140-6736(20)30361-5

30. Gu J, Han B, Wang J. COVID-19: Gastrointestinal Manifestations and Potential Fecal-Oral Transmission [published online ahead of print, 2020 Mar 3].
Gastroenterology. 2020. https://doi.org/10.1053/j.gastro.2020.02.054

31. Chen L, Lou J, Bai Y, Wang M. COVID-19 Disease with Positive Fecal and Negative Pharyngeal and Sputum Viral Tests [published online ahead of print, 2020 Mar 20]. Am J Gastroenterol. 2020. https://doi.org/10.14309/ ajg.0000000000000610

32. Wölfel R, Corman VM, Guggemos W, Seilmaier M, Zange S, Müller MA, et al. Virological assessment of hospitalized patients with COVID-2019. Nature. 2020. https:// doi,org/10.1038/s41586-020-2196-x

33. Sultan S, Lim JK, Altayar O, Davitkov P, Feuerstein JD, Siddique SM, et al. AGA Institute Rapid Recommendations for Gastrointestinal Procedures During the COVID19 Pandemic. Gastroenterology. 2020. https://doi. org/10.1053/j.gastro.2020.03.072

34. Repici A, Maselli R, Colombo M, Spadaccini M, Anderloni A, Carrara S, et al. Coronavirus (COVID-19) outbreak: what the department of endoscopy should know. Gastrointest Endosc. 2020. https://doi.org/10.1016/j.gie.2020.03.019

35. Sociedad Española de Endoscopia Digestiva (SEED). Recomendaciones de la SEED: Protección en Unidades de Endoscopia frente al COVID-19 [Internet] [acceso 15 de abril de 2020]. Disponible en: https://wseed.es/ images/site/guia_clinica/2020/RecomendacionesSEED_ ProteccionUnidadesEndoscopia_Coronavirus.pdf

36. Zhang Y, Zhang X, Liu L, Wang $\bar{H}$, Zhao Q. Suggestions for infection prevention and control in digestive endoscopy during current 2019-nCoV pneumonia outbreak in Wuhan, Hubei province, China. Endoscopy. 2020;52(4):312-314. https://doi.org/10.1055/a-1128-4313

37. Otero W, Gómez M, Ángel LA, Ruiz O, Marulanda $\mathrm{H}$, Riveros J, et al. Procedimientos endoscópicos y pandemia COVID-19 Consideraciones básicas. Rev Colomb Gastroenterol. 2020;35(1):65-75. https://doi. org/10.22516/25007440.526

38. Rubin DT, Feuerstein JD, Wang AY, Cohen RD. AGA Clinical Practice Update on Management of Inflammatory Bowel Disease During the COVID-19 Pandemic: Expert Commentary. Gastroenterology. 2020. https://doi. org/10.1053/j.gastro.2020.04.012 